\title{
Sputum eosinophilia can predict responsiveness to inhaled corticosteroid treatment in patients with overlap syndrome of COPD and asthma
}

This article was published in the following Dove Press journal:

International Journal of COPD

II April 2012

Number of times this article has been viewed

\section{Yoshiaki Kitaguchi ${ }^{1, *}$ \\ Yoshimichi Komatsu',* \\ Keisaku Fujimoto² \\ Masayuki Hanaoka' \\ Keishi Kubo'}

'First Department of Internal Medicine, Shinshu University School of Medicine, ${ }^{2}$ Department of Biomedical Laboratory Sciences, Shinshu University School of Health Sciences, Matsumoto, Japan

*These authors contributed equally to this work
Correspondence: Keisaku Fujimoto Department of Biomedical Laboratory Sciences, Shinshu University School of Health Sciences, 3-I-I Asahi, Matsumoto 390-8621, Japan

$\mathrm{Tel}+8 \mid 263372393$

Fax +8I 263372370

Email keisaku@shinshu-u.ac.jp
Background: Chronic obstructive pulmonary disease (COPD) and asthma may overlap and converge in older people (overlap syndrome). It was hypothesized that patients with overlap syndrome may have different clinical characteristics such as sputum eosinophilia, and better responsiveness to treatment with inhaled corticosteroid (ICS).

Methods: Sixty-three patients with stable COPD (forced expiratory volume in 1 second $\left[\mathrm{FEV}_{1}\right] \leq 80 \%$ ) underwent pulmonary function tests, including reversibility of airflow limitation, arterial blood gas analysis, analysis of inflammatory cells in induced sputum, and chest highresolution computed tomography. The inclusion criteria for COPD patients with asthmatic symptoms included having asthmatic symptoms such as episodic breathlessness, wheezing, cough, and chest tightness worsening at night or in the early morning (COPD with asthma group). The clinical features of COPD patients with asthmatic symptoms were compared with those of COPD patients without asthmatic symptoms (COPD without asthma group).

Results: The increases in $\mathrm{FEV}_{1}$ in response to treatment with ICS were significantly higher in the COPD with asthma group. The peripheral eosinophil counts and sputum eosinophil counts were significantly higher. The prevalence of patients with bronchial wall thickening on chest high-resolution computed tomography was significantly higher. A significant correlation was observed between the increases in FEV in response to treatment with ICS and sputum

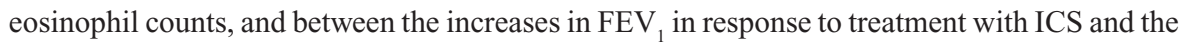
grade of bronchial wall thickening. Receiver operating characteristic curve analysis revealed $82.4 \%$ sensitivity and $84.8 \%$ specificity of sputum eosinophil count for detecting COPD with asthma, using $2.5 \%$ as the cutoff value.

Conclusion: COPD patients with asthmatic symptoms had some clinical features. ICS should be considered earlier as a potential treatment in such patients. High sputum eosinophil counts and bronchial wall thickening on chest high-resolution computed tomography might therefore be a good predictor of response to ICS.

Keywords: COPD, asthma, HRCT, inhaled corticosteroid, pulmonary function

\section{Introduction}

Chronic obstructive pulmonary disease (COPD) is a disease state characterized by airflow limitation that is not fully reversible according to the Global Initiative for Chronic Obstructive Lung Disease guidelines. ${ }^{1}$ Irreversible airflow limitation is defined as the proportion of forced vital capacity (FVC) in 1 second, ie, forced expiratory volume in 1 second $\left(\mathrm{FEV}_{1}\right) / \mathrm{FVC}$ ratio, being $<70 \%$ after inhalation of $\beta_{2}$-agonist. However, COPD is not a simple homogenous disease clinically defined only as irreversible airflow limitation. For example, COPD has some morphological phenotypes on chest high-resolution computed tomography (HRCT), and each phenotype 
is associated with clinical characteristics and different responsiveness to bronchodilators. ${ }^{2,3}$ Moreover, COPD can coexist with asthma which may also cause irreversible airflow limitation, ${ }^{4}$ although COPD and asthma are two quite different and independent diseases. Asthma may be a risk factor for the development of COPD. ${ }^{5}$ In particular, COPD and asthma may overlap and converge in older people. ${ }^{6,7}$ However, the potential for overlap of the individual obstructive airway disease syndromes, including COPD and asthma, has received less attention. ${ }^{8}$

For this study, it was hypothesized that COPD patients with asthmatic symptoms may have different clinical features than COPD patients without asthmatic symptoms. The aim of this study was to clarify the features of COPD patients with asthmatic symptoms, and these findings may therefore play a role in developing the optimal strategy for the management of COPD with asthmatic symptoms.

\section{Methods}

\section{Subjects}

A total of 63 patients with stable COPD, who showed $\mathrm{FEV}_{1} / \mathrm{FVC}<70 \%$ and $\mathrm{FEV}_{1}<80 \%$ of the predicted value after inhalation of a $\beta_{2}$-agonist (moderate to very severe COPD) and were seen at the outpatient clinic of Shinshu University Hospital (Matsumoto, Japan) from February 2007 to July 2009, were recruited for this study. The diagnosis of COPD was based on the clinical history and symptoms, including dyspnea on exertion and pulmonary function characterized by irreversible airflow limitation $\left(\mathrm{FEV}_{1} / \mathrm{FVC}<70 \%\right.$ after inhalation of a $\beta_{2}$-agonist $)$ in accordance with the Global Initiative for Chronic Obstructive Lung Disease guidelines. ${ }^{1}$ All subjects had smoking-related COPD without $\alpha 1$-antitrypsin deficiency, and had a smoking history of more than 30 pack years. All patients with COPD without asthmatic symptoms had no history of asthma or asthmatic symptoms (COPD without asthma group). All patients with COPD with asthmatic symptoms had experienced asthmatic symptoms such as episodic breathlessness, wheezing, cough, and chest tightness worsening at night or in the early morning (COPD with asthma group). The exclusion criteria included the presence of a respiratory tract infection or exacerbation of COPD and/or asthma during the preceding 3 months. In total, 46 patients were in the COPD without asthma group and 17 patients were in the COPD with asthma group. Most of the patients used short-acting $\beta_{2}$-agonists as needed to relieve dyspnea two to three times per week, but did not use them on the day that the pulmonary function tests were performed.
The study was approved by the institutional research ethics committee of Shinshu University School of Medicine (Matsumoto, Japan), and all patients gave written informed consent to participate.

\section{Protocol}

Information was obtained from each patient on the history of the current illness including complications and history of smoking, physical and laboratory examinations, pulmonary function tests including reversibility of airflow limitation by $\beta_{2}$-agonist ( $20 \mu \mathrm{g}$ of inhaled procaterol hydrochloride), arterial blood gas analysis, analysis of inflammatory cells in induced sputum, and the findings on chest HRCT. The patients were treated with inhaled corticosteroid (ICS; $400 \mu \mathrm{g}$ /day of inhaled fluticasone propionate) for 2-3 months. Pulmonary measurements were again obtained by spirometry after treatment.

\section{Pulmonary function tests}

Spirometry and measurements of the diffusing capacity for carbon monoxide (DLCO) were performed using a pulmonary function testing system (Chestac-55V; Chest Co, Ltd, Tokyo, Japan). $\mathrm{FEV}_{1}$ was measured before and 20 minutes after inhalation of $\beta_{2}$-agonist ( $20 \mu \mathrm{g}$ of procaterol hydrochloride) by aerosol (metered-dose inhaler) with a spacer (Meptin ${ }^{\circledR}$; Otsuka Pharmaceutical, Tokushima, Japan) to evaluate the reversibility of airflow limitation. The functional residual capacity was measured using a body box (MedGraphics model 1085; Medical Graphics Corp, Minneapolis, MN), after which the subjects immediately inspired to total lung capacity and expired maximally to residual volume, thus allowing calculation of lung volume and residual volume/total lung capacity. The pulmonary function tests were performed by two special technicians according to the American Thoracic Society criteria. Two or three tests were repeated to guarantee repeatability.

\section{Sputum collection and analysis}

Sputum induced by the inhalation of hypertonic saline was collected as described previously. ${ }^{9}$ Briefly, all subjects inhaled a $\beta_{2}$-agonist and $3.5 \%$ hypertonic saline nebulized with an ultrasonic nebulizer (NE-V10B; Omron Corporation, Tokyo, Japan). The nebulization was continued for at least 10 minutes and stopped after 15 minutes or earlier if a $\geq 2 \mathrm{~mL}$ sputum sample of good quality was obtained. The collected sputum was then separated from contaminating saliva by macroscopic examination, and any mucus plugs were removed from the dish to a sterile plastic container, after which the volume of the sample was determined. The sample was incubated with an equal volume of Hank's balanced salt solution containing $1 \mathrm{mM}$ dithiothreitol (Sigma-Aldrich Company 
Ltd, Poole, United Kingdom) at $37^{\circ} \mathrm{C}$ for 15 minutes. The residual mucous was removed and the eluent was used to obtain total and differential cell counts. The total cell count, except that of squamous cells, was determined with a standard hemocytometer (Fuchs Rosenthal; Erma, Tokyo, Japan), normalized for weight and expressed as cells $10^{5} / \mathrm{g}$ wet weight of sputum. Cell smears were prepared with a centrifuge (Autosmear ${ }^{\text {TM}}$; Sakura Keishi Kabushiki Kaisha, Tokyo, Japan) and stained with May-Grünwald-Giemsa (Merck, Darmstadt, Germany) for differential cell counting. The slides were coated and then 500 cells were counted to determine the differential leukocyte count.

\section{HRCT protocol and evaluation of the degree of emphysema and bronchial wall thickening (BWT)}

A helical CT scanner (LightSpeed VCT; GE Healthcare, Waukesha, WI) was used for conventional contiguous scanning with a slice thickness of $10 \mathrm{~mm}$ to screen for chest abnormalities followed by HRCT scanning at full inspiration (at total lung capacity level) with $1 \mathrm{~mm}$ collimation $(120 \mathrm{kVp}, 200 \mathrm{~mA}$, pitch 1.0). Four $1 \mathrm{~mm}$ thick slices were obtained at three anatomical levels at full inspiration: near the superior margin of the aortic arch, at the level of the carina, and at the level of the orifice of the inferior pulmonary veins. The low attenuation area (LAA) was visually scored in each bilateral lung field according to the method of Goddard et al. ${ }^{10}$ Total scores were calculated and the severity of emphysema was graded as: score 0 , LAA $<5 \%$; score $1,5 \% \leq$ LAA $<25 \%$; score $2,25 \% \leq$ LAA $<50 \%$; score $3,50 \% \leq$ LAA $<75 \%$; and score 4 , LAA $\geq 75 \%$. The severity of emphysema was graded in accordance with the sum of the scores for six dimensions: grade 0 , total score $=0$; grade 1 , total score $=1-6$; grade 2 , total score $=7-12$; grade 3, total score $=13-18$; and grade 4 , total score $=19-24$. The patients were classified according to the visual HRCT findings as: absence of emphysema phenotype, which showed little emphysema and at least LAA grade 1, and emphysema phenotype, which showed apparent emphysema (at least grade 2). BWT in all lung fields was graded visually as reported previously: ${ }^{11,12}$ grade 0 , none; grade $1,<50 \%$ adjacent pulmonary artery diameter; and grade $2, \geq 50 \%$ adjacent pulmonary artery diameter. The patients without BWT showed BWT of grade 0 , and patients with BWT showed BWT of more than grade 1 . HRCT images were analyzed independently by two pulmonologists with no knowledge of the patients' clinical status.

\section{Statistical analysis}

The values shown in the text and tables are the mean \pm standard error of the mean. The data distribution of the variables in the groups was first assessed with Bartlett's test. Data for the variables that showed a normal distribution were compared using the parametric Student's $t$-test. Data for the variables that did not show a normal distribution were compared using the nonparametric Mann-Whitney U-test. Simple correlations between variables were examined by calculating Pearson's product correlation coefficient. Cutoff values of the sputum eosinophil count for detecting COPD with asthma were calculated from receiver operator characteristic curve analysis, with sensitivity and specificity determined in each case. All statistical analyses were performed with the use of a Windows ${ }^{\circledR}$-compatible software program (StatFlex version 5.0; Artech Ltd, Osaka, Japan). A $P$ value of less than 0.05 was considered to be significant in all statistical analyses.

\section{Results}

\section{Clinical characteristics of each group}

There were no significant differences in age, gender, body mass index, Brinkman index, history of sinusitis, or history of noxious particles or gases other than tobacco between the two groups (Table 1). The prevalence of history of allergic rhinitis was significantly higher in the COPD with asthma group. A laboratory analysis showed no cases of $\alpha_{1}$-antitrypsin deficiency, and no significant difference was observed in the serum $\alpha_{1}$-antitrypsin and serum total immunoglobulin E levels between the two groups. The peripheral eosinophil counts were significantly higher in the COPD with asthma group. Seven of the COPD patients

Table I Clinical characteristics and laboratory data in patients with chronic obstructive pulmonary disease with and without asthma

\begin{tabular}{|c|c|c|}
\hline & $\begin{array}{l}\text { COPD without } \\
\text { asthma } \\
(n=46)\end{array}$ & $\begin{array}{l}\text { COPD with } \\
\text { asthma } \\
(n=17)\end{array}$ \\
\hline Age (years) & $71 \pm 1$ & $73 \pm 1$ \\
\hline Gender (female/male) & $6 / 40$ & $0 / 17$ \\
\hline Body mass index $\left(\mathrm{kg} / \mathrm{m}^{2}\right)$ & $20.6 \pm 0.5$ & $21.3 \pm 0.8$ \\
\hline History of smoking (pack years) & $60.8 \pm 4.1$ & $55.5 \pm 5.4$ \\
\hline History of sinusitis ( $[\%]$ ) & $9(19.7 \%)$ & 5 (29.4\%) \\
\hline History of allergic rhinitis & $4(8.7 \%)$ & $5(29.4 \%)^{*}$ \\
\hline $\begin{array}{l}\text { History of noxious particles or } \\
\text { gases other than tobacco }(\mathrm{n}[\%])\end{array}$ & $18(39.1 \%)$ & 7 (4I.2\%) \\
\hline$\alpha \mathrm{I}$-antitrypsin (mg/dL) & $150.0 \pm 8.6$ & $136.4 \pm 4.7$ \\
\hline $\begin{array}{l}\text { Serum total immunoglobulin } \mathrm{E} \\
(\mathrm{IU} / \mathrm{mL})^{\ddagger}\end{array}$ & $249.0 \pm 99.4$ & $693.1 \pm 309.4$ \\
\hline Peripheral eosinophil count $\left(/ \mathrm{mm}^{3}\right)$ & $207.9 \pm 31.7$ & $407.5 \pm 81.8^{*}$ \\
\hline
\end{tabular}

Notes: Values are the number (\%) or the mean \pm standard error of the mean; $* P<0.05$ versus chronic obstructive pulmonary disease without asthma; ${ }^{\ddagger}$ normal range is $<400 \mathrm{IU} / \mathrm{mL}$.

Abbreviation: COPD, chronic obstructive pulmonary disease. 
with asthmatic symptoms (41.2\%) had a history of exposure to noxious particles or gases other than tobacco; three patients $(17.6 \%)$ to asbestos and four patients $(23.5 \%)$ to agrochemical compounds. Eighteen of the COPD patients without asthmatic symptoms (39.1\%) had a history of exposure to noxious particles or gases other than tobacco; eight patients $(17.4 \%)$ to asbestos and ten patients $(21.7 \%)$ to agrochemical compounds.

\section{Pulmonary function tests and inflammatory cell analysis of induced sputum}

There were no significant differences in the vital capacity, $\mathrm{FEV}_{1}$, or $\mathrm{FEV}_{1} / \mathrm{FVC}$ (Table 2). There were no significant differences in lung hyperinflation expressed by increased residual volume and total lung capacity between the two groups. DLCO was significantly higher in the COPD with asthma group. An arterial blood gas analysis showed no significant differences between the two groups.

There was no significant difference in the increases in $\mathrm{FEV}_{1}$ in response to $\beta_{2}$-agonist between the two groups (Table 3). The increases in $\mathrm{FEV}_{1}$ in response to treatment with ICS were significantly higher in the COPD with asthma group. Twelve patients in the COPD without asthma group $(26.1 \%)$ and eleven patients in the COPD with asthma group (64.7\%) showed a reversibility of airflow limitation, which was defined as an increase in $\mathrm{FEV}_{1}$ of $>12 \%$ and $200 \mathrm{~mL}$ from baseline values, in response to treatment with ICS for 2-3 months, and none of the patients showed $\mathrm{FEV}_{1} / \mathrm{FVC} \geq 70 \%$ following the treatment with ICS.

Table 2 Pulmonary function in patients with chronic obstructive pulmonary disease with and without asthma

\begin{tabular}{lcc}
\hline & $\begin{array}{l}\text { COPD without asthma } \\
(\mathbf{n}=\mathbf{4 6})\end{array}$ & $\begin{array}{l}\text { COPD with asthma } \\
(\mathbf{n}=17)\end{array}$ \\
\hline $\mathrm{VC}$ (\% pred) & $92.3 \pm 3.1$ & $96.6 \pm 3.6$ \\
$\mathrm{FEV}$ (\% pred) & $47.5 \pm 2.8$ & $51.3 \pm 3.5$ \\
$\mathrm{FEV} / \mathrm{FVC}(\%)$ & $46.1 \pm 1.7$ & $50.9 \pm 2.9$ \\
$\mathrm{TLC}(\%$ pred) & $132.0 \pm 3.3$ & $120.6 \pm 4.9$ \\
$\mathrm{RV}(\%$ pred) & $228.5 \pm 9.9$ & $192.8 \pm 13.9$ \\
$\mathrm{RV} / \mathrm{TLC}(\%)$ & $57.0 \pm 1.5$ & $51.7 \pm 1.9$ \\
$\mathrm{DLCO}(\%$ pred) & $56.2 \pm 3.5$ & $72.2 \pm 5.4^{*}$ \\
$\mathrm{PaO}_{2}$ (Torr) & $67.7 \pm 1.8$ & $75.9 \pm 2.7$ \\
$\mathrm{PaCO}_{2}$ (Torr) & $42.0 \pm 0.8$ & $40.4 \pm 1.0$ \\
\hline
\end{tabular}

Notes: Values are the number (\%) or the mean \pm standard error of the mean; $* P<0.05$ versus chronic obstructive pulmonary disease without asthma.

Abbreviations: COPD, chronic obstructive pulmonary disease; DLCO, diffusing capacity for carbon monoxide; $\mathrm{FEV}_{1}$, forced expiratory volume in I second; FVC, forced vital capacity; $\mathrm{PaO}_{2}$, partial pressure of arterial oxygen; $\mathrm{PaCO}_{2}$, partial pressure of arterial carbon dioxide; RV, residual volume; TLC, total lung capacity; VC, vital capacity; \% pred, percent predicted.
Table 3 Responses to $\beta_{2}$-agonist and inhaled corticosteroid, and cell analysis in induced sputum

\begin{tabular}{|c|c|c|}
\hline & $\begin{array}{l}\text { COPD without } \\
\text { asthma } \\
(n=46)\end{array}$ & $\begin{array}{l}\text { COPD with } \\
\text { asthma } \\
(n=I 7)\end{array}$ \\
\hline \multicolumn{3}{|l|}{ Response to $\beta_{2}$-agonist } \\
\hline$\Delta \mathrm{FEV}_{1}(\mathrm{~mL})$ & $123.7 \pm 17.6$ & $186.5 \pm 29.8$ \\
\hline$\%$ change in $\mathrm{FEV}_{1}(\%)$ & $11.4 \pm 2.0$ & $17.2 \pm 3.8$ \\
\hline \multicolumn{3}{|c|}{ Response to treatment with inhaled corticosteroid } \\
\hline$\Delta \mathrm{FEV},(\mathrm{mL})$ & $120.2 \pm 21.5$ & $372.4 \pm 58.0 * *$ \\
\hline$\%$ change in $\mathrm{FEV}_{1}(\%)$ & $12.8 \pm 2.2$ & $32.4 \pm 6.2^{* *}$ \\
\hline \multicolumn{3}{|l|}{ Sputum cell differentiation } \\
\hline Total cell $\left(\times 10^{5} / g\right)$ & $87.5 \pm 24.8$ & $109.3 \pm 25.4$ \\
\hline Macrophage (\%) & $11.6 \pm 1.8$ & $9.9 \pm 2.2$ \\
\hline Lymphocyte (\%) & $3.8 \pm 0.5$ & $3.8 \pm 0.7$ \\
\hline Neutrophil (\%) & $82.6 \pm 2.1$ & $74.0 \pm 4.4$ \\
\hline Eosinophil (\%) & $2.0 \pm 0.5$ & $12.3 \pm 3.3 * *$ \\
\hline
\end{tabular}

Notes: Values are the number (\%) or the mean \pm standard error of the mean; **P $<0.01$ versus chronic obstructive pulmonary disease without asthma. Abbreviations: COPD, chronic obstructive pulmonary disease; $\mathrm{FEV}_{1}$, forced expiratory volume in I second; $\triangle \mathrm{FEV}_{\mathrm{l}}$, increases in forced expiratory volume in I second.

There was no significant difference in the total cell counts in induced sputum. However, the eosinophil counts in induced sputum were significantly higher in the COPD with asthma group. Figure 1 shows the relationship between the increases in $\mathrm{FEV}_{1}$ in response to treatment with ICS and sputum eosinophil counts. A significant correlation was found $(r=0.42$, $P=0.0006)$. Receiver operator characteristic curve analysis was performed to determine the sputum eosinophil count for detecting COPD with asthma. The sensitivity and specificity of the sputum eosinophil count for detecting COPD with asthma were $82.4 \%$ and $84.8 \%$, respectively, when the cutoff value for the sputum eosinophil count was $2.5 \%$.

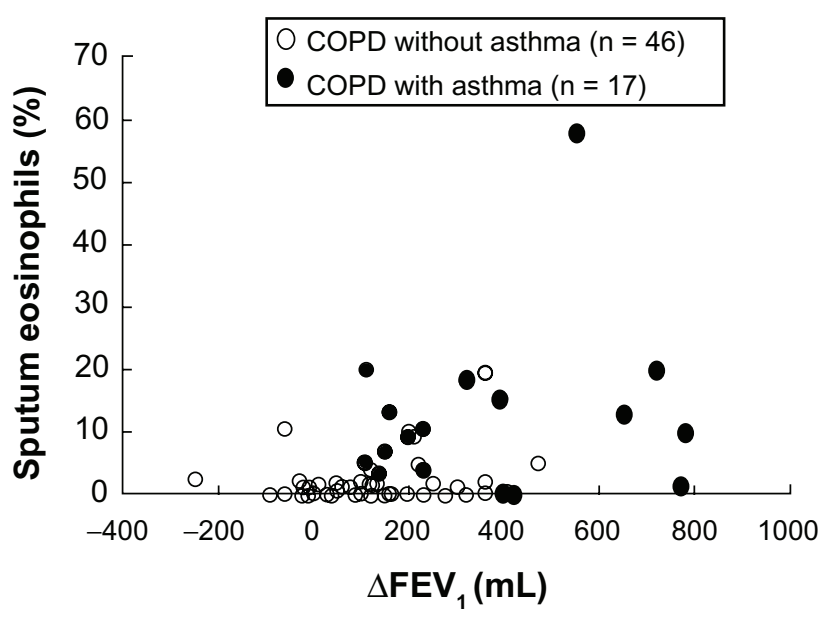

Figure I Relationship between response to treatment of inhaled corticosteroid and sputum eosinophils counts.

Abbreviations: COPD, chronic obstructive pulmonary disease; $\triangle \mathrm{FEV}$, , increases in forced expiratory volume in I second. 


\section{Degree of emphysema and BWT according to the findings of HRCT}

No significant difference was found in the score of LAA between the two groups (Table 4). Thirty-eight of 46 patients in the COPD without asthma group and 13 of 17 patients in the COPD with asthma group were classified into the emphysema phenotype. The remaining eight patients in the COPD without asthma group and four patients in the COPD with asthma group were classified into the absence of emphysema phenotype. The prevalence of patients with BWT was significantly higher in the COPD with asthma group. Furthermore, the prevalence of patients with BWT was significantly higher in the COPD with asthma group than in the COPD without asthma group only in the patients with the emphysema phenotype. A significant correlation was observed between the increases in $\mathrm{FEV}_{1}$ in response to treatment with ICS and the grade of BWT $(\mathrm{r}=0.41, P=0.0007)$.

\section{Discussion}

The current study found the increases in $\mathrm{FEV}_{1}$ in response to treatment with ICS were significantly higher in the COPD with asthma group, although no significant difference was found in the increases in $\mathrm{FEV}_{1}$ in response to $\beta_{2}$-agonist between the two groups. Both the peripheral eosinophil counts and sputum eosinophil counts were significantly higher in the COPD with asthma group. Chest HRCT showed that the prevalence of patients with BWT was significantly higher in the COPD with asthma group. A significant correlation was observed between the increases in $\mathrm{FEV}_{1}$ in response to treatment with ICS and sputum eosinophil counts, and between the increases in $\mathrm{FEV}_{1}$ in response to treatment with ICS and the grade of BWT. Receiver operator characteristic

Table 4 Chest high-resolution computed tomography findings

\begin{tabular}{lll}
\hline & $\begin{array}{l}\text { COPD without } \\
\text { asthma } \\
(\mathbf{n}=\mathbf{4 6})\end{array}$ & $\begin{array}{l}\text { COPD with } \\
\text { asthma } \\
(\mathbf{n}=17)\end{array}$ \\
\hline LAA score & $17.9 \pm 1.3$ & $13.8 \pm 2.3$ \\
BWT (+) (n [\%]) & $16(34.8 \%)$ & $1 \mathrm{I}(64.7 \%)^{*}$ \\
Constitution of the phenotypes & $38(82.6 \%)$ & $13(76.5 \%)$ \\
Emphysema phenotype & $26(56.5 \%)$ & $4(23.5 \%)^{*}$ \\
BWT (-) & $12(26.1 \%)$ & $9(52.9 \%)^{*}$ \\
BWT (+) & $8(17.4 \%)$ & $4(23.5 \%)$ \\
Absence of emphysema phenotype & $4(8.7 \%)$ & $2(11.8 \%)$ \\
BWT (-) & $4(8.7 \%)$ & $2(11.8 \%)$ \\
BWT (+)
\end{tabular}

Notes: Values are the number (\%) or the mean \pm standard error of the mean; $* P<0.05$ versus chronic obstructive pulmonary disease without asthma.

Abbreviations: BWT (-), bronchial wall thickening of grade 0; BWT (+), bronchial wall thickening of more than grade I; COPD, chronic obstructive pulmonary disease; LAA, low attenuation area. curve analysis revealed $82.4 \%$ sensitivity and $84.8 \%$ specificity of sputum eosinophil count for detecting COPD with asthma, using $2.5 \%$ as the cutoff value.

The underlying chronic airway inflammation in COPD is very different than that in asthma. Asthma is traditionally characterized by an eosinophilic inflammation that affects all the airways but not lung parenchyma, and it is linked to airway hyperresponsiveness. ${ }^{13}$ However, asthmatic patients that smoke develop pathological features similar to COPD. ${ }^{14}$ Some patients with COPD may demonstrate features of asthma, such as a mixed inflammatory pattern with increased eosinophils, ${ }^{15}$ and an increased sputum eosinophil count has been reported to be related to an improvement in $\mathrm{FEV}_{1}$ following treatment with ICS in COPD. ${ }^{16}$ The peripheral eosinophil counts and sputum eosinophil counts were significantly higher, and the reversibility due to a response to the treatment with ICS was better in the COPD with asthma group in the current series. These results suggest that COPD patients with asthmatic symptoms also had features of asthma such as a mixed inflammatory pattern with increased eosinophils. A significant correlation was observed between the increases in $\mathrm{FEV}_{1}$ in response to treatment with ICS and sputum eosinophil counts, thus suggesting that high sputum eosinophil counts might be a good predictor of response to ICS.

Pulmonary function testing with bronchodilators has been used to differentiate asthma from COPD, with COPD demonstrating a smaller response.${ }^{17}$ However, some patients with COPD showed reversibility, which is defined as an increase in $\mathrm{FEV}_{1}$ of $>12 \%$ and $200 \mathrm{~mL}$ from baseline values, in response to bronchodilators. ${ }^{18}$ The degree of reversibility of airflow limitation is no longer recommended for diagnosis, differential diagnosis with asthma, or predicting the response to longer treatment with bronchodilators or glucocorticosteroids according to the Global Initiative for Chronic Obstructive Lung Disease guidelines. ${ }^{1}$ The current study found no significant difference in the increases in $\mathrm{FEV}_{1}$ in response to $\beta_{2}$-agonist between the two groups, and this result was consistent with the findings of previous reports.

The major differences in airway remodeling between asthma and COPD are the thickening of the reticular layer under the basement membrane with submucosal infiltration of a large number of eosinophils and the proliferation of mucosal blood vessels found in asthma. On the contrary, it seems that fixed airway obstruction is the final result of structural changes of peripheral airways and lung parenchyma in COPD. ${ }^{13,19,20}$ Therefore, these diseases have different pathologies of airway remodeling. However, it is difficult to clinically differentiate between COPD and asthma in some patients with irreversible 
airflow limitation. ${ }^{13}$ Airway remodeling is thought to be the main cause of irreversible airflow limitation in older asthmatic patients. The presence of a normal diffusing capacity for DLCO may be useful to differentiate asthma with airway remodeling from COPD. However, COPD has morphological phenotypes on chest HRCT and some patients with COPD that show normal capacity for DLCO are classified into the absence of emphysema phenotype. ${ }^{2,3}$ There is a possibility that the COPD with asthma group included asthmatic patients with airway remodeling in this study, because it is sometimes difficult to clinically differentiate such patients from COPD patients who are classified into the absence of emphysema phenotype. Nonetheless, ICS should be considered earlier as a potential treatment in patients who are clinically diagnosed to have COPD with asthmatic symptoms. While DLCO was significantly lower in the COPD with asthma group, this may be because the mean value of LAA score was lower in the COPD with asthma group. Alternatively, this may be because asthmatic patients show relatively higher values of DLCO because allergic inflammation may affect pulmonary circulation. $^{21}$

HRCT used to quantify abnormalities of the airways due to airway remodeling in asthma, and the HRCT scan score is correlated with the severity of asthma and airflow obstruction..$^{22-24}$ The absence of emphysema phenotype and emphysema with BWT phenotype are associated with a better responsiveness to treatment with ICS in COPD patients. ${ }^{2,3}$ The prevalence of patients with BWT was significantly higher in the COPD with asthma group on chest HRCT in the current series. A significant correlation was observed between the increases in $\mathrm{FEV}_{1}$ in response to treatment with ICS and the grade of BWT. The current results were consistent with those of previous reports and suggest that BWT on chest HRCT might be a good predictor of response to ICS.

One limitation of this retrospective study is that the assessment of emphysema was done by a visual scoring method, rather than using a software-based quantification of emphysema. However, the reproducibility of such visual scoring has been demonstrated in a previous report. ${ }^{3}$ Another limitation is the lack of statistical power because the sample size was small for the absence of emphysema phenotype ( $n=4$ in the COPD with asthma group and $n=8$ in the COPD without asthma group) which is generally lower, accounting for only about $22 \%$ of COPD. ${ }^{2,3}$ Another limitation of these findings is associated with the fact that while many statistical differences and correlations were observed, these results by themselves do not imply cause and effect.

\section{Conclusion}

COPD patients with asthmatic symptoms had some clinical features such as high peripheral eosinophil counts, high sputum eosinophil counts, preserved diffusing capacity, high prevalence of BWT on chest HRCT, and better reversibility responsive to treatment with ICS. ICS should thus be considered earlier as a potential treatment in COPD patients with asthmatic symptoms. High sputum eosinophil counts and BWT on chest HRCT might therefore be a good predictor of response to ICS.

\section{Disclosure}

The authors report no conflicts of interest in this work.

\section{References}

1. Global Initiative for Chronic Obstructive Lung Disease. Global strategy for the diagnosis, management, and prevention of chronic obstructive pulmonary disease. Updated 2010. Available from: http://www. goldcopd.org/Guidelines/guideline-2010-gold-report.html. Accessed February 7, 2012.

2. Kitaguchi Y, Fujimoto K, Kubo K, Honda T. Characteristics of COPD phenotypes classified according to the findings of HRCT. Respir Med. 2006;100(10):1742-1752.

3. Fujimoto K, Kitaguchi Y, Kubo K, Honda T. Clinical analysis of chronic obstructive pulmonary disease phenotypes classified using high-resolution computed tomography. Respirology. 2006;11(6): 731-740.

4. Vonk JM, Jongepier H, Panhuysen CI, Schouten JP, Bleecker ER, Postma DS. Risk factors associated with the presence of irreversible airflow limitation and reduced transfer coefficient in patients with asthma after 26 years of follow up. Thorax. 2003;58(4):322-327.

5. Silva GE, Sherrill DL, Guerra S, Barbee RA. Asthma as a risk factor for COPD in a longitudinal study. Chest. 2004;126(1):59-65.

6. Diaz-Guzman E, Mannino DM. Airway obstructive diseases in older adults: from detection to treatment. J Allergy Clin Immunol. 2010;126(4):702-709.

7. Gibson PG, McDonald VM, Marks GB. Asthma in older adults. Lancet. 2010;376(9743):803-813.

8. Gibson PG, Simpson JL. The overlap syndrome of asthma and COPD: what are its features and how important is it? Thorax. 2009;64(8):728-735.

9. Fujimoto K, Kubo K, Matsuzawa Y, Sekiguchi M. Eosinophil cationic protein levels in induced sputum correlate with the severity of bronchial asthma. Chest. 1997;112(5):1241-1247.

10. Goddard PR, Nicholson EM, Laszlo G, Watt I. Computed tomography in pulmonary emphysema. Clin Radiol. 1982;33(4):379-387.

11. Roberts HR, Wells AU, Milne DG, et al. Airflow obstruction in bronchiectasis: correlation between computed tomography features and pulmonary function tests. Thorax. 2000;55(3):198-204.

12. Copley SJ, Wells AU, Muller NL, et al. Thin-section CT in obstructive pulmonary disease: discriminatory value. Radiology. 2002;223(3): 812-819.

13. Tzortzaki EG, Proklou A, Siafakas NM. Asthma in the elderly: can we distinguish it from COPD? J Allergy (Cairo). 2011;2011:843543.

14. Thomson NC, Chaudhuri R, Livingston E. Asthma and cigarette smoking. Eur Respir J. 2004;24(5):822-833.

15. Fujimoto K, Kubo K, Yamamoto H, Yamaguchi S, Matsuzawa Y. Eosinophilic inflammation in the airway is related to glucocorticoid reversibility in patients with pulmonary emphysema. Chest. 1999; 115(3):697-702. 
16. Brightling CE, McKenna S, Hargadon B, et al. Sputum eosinophilia and the short term response to inhaled mometasone in chronic obstructive pulmonary disease. Thorax. 2005;60(3):193-198.

17. Global Initiative for Asthma (GINA). Global strategy for asthma management and prevention. Updated 2010. Available from: http://www.ginasthma.org/guideline-report-2010.html. Accessed February 7, 2012.

18. Tashkin DP, Celli B, Decramer M, et al. Bronchodilator responsiveness in patients with COPD. Eur Respir J. 2008;31(4):742-750.

19. Jeffery PK. Remodeling and inflammation of bronchi in asthma and chronic obstructive pulmonary disease. Proc Am Thorac Soc. 2004;1(3):176-183.

20. Jeffery PK. Remodeling in asthma and chronic obstructive lung disease. Am J Respir Crit Care Med. 2001;164(10 Pt 2):S28-S38.
21. Collard P, Njinou B, Nejadnik B, Keyeux A, Frans A. Single breath diffusing capacity for carbon monoxide in stable asthma. Chest. 1994;105(5):1426-1429.

22. Nakano Y, Muller NL, King GG, et al. Quantitative assessment of airway remodeling using high-resolution CT. Chest. 2002;122(Suppl 6): 271S-275S.

23. Little SA, Sproule MW, Cowan MD, et al. High resolution computed tomographic assessment of airway wall thickness in chronic asthma: reproducibility and relationship with lung function and severity. Thorax. 2002;57(3):247-253.

24. Vignola AM, Paganin F, Capieu L, et al. Airway remodelling assessed by sputum and high-resolution computed tomography in asthma and COPD. Eur Respir J. 2004;24(6):910-917.

\section{Publish your work in this journal}

The International Journal of COPD is an international, peer-reviewed journal of therapeutics and pharmacology focusing on concise rapid reporting of clinical studies and reviews in COPD. Special focus is given to the pathophysiological processes underlying the disease, intervention programs, patient focused education, and self management protocols.

\section{Dovepress}

This journal is indexed on PubMed Central, MedLine and CAS. The manuscript management system is completely online and includes a very quick and fair peer-review system, which is all easy to use. Visit http://www.dovepress.com/testimonials.php to read real quotes from published authors. 